LBL -32021

DE92 041172

\title{
Alternative Formulations of the \\ Laplace Transform Boundary Element (LTBE) Numerical Method for the Solution of Diffusion-Type Equations
}

\author{
George Moridis \\ Earth Sciences Division \\ Lawrence Berkeley Laboratory \\ University of California \\ Berkeley, California 94720
}

March 1992

This work was supported by the U.S. Department of Energy under Contract No. DE-AC03-76SF00098. 


\section{Alternative Formulations of The Laplace Transform Boundary Element (LTBE) Numerical Method for the Solution of Diffusion-Type Equations} G. J. Moridis Earth Sciences Division, Lawrence Berkeley Laboratory University of California, Berkeley, CA 94720, U.S.A.

\section{ABSTRACT}

The Laplace Transform Boundary Element (LTBE) method is a recently introduced numerical method, and has been used for the solution of diffusion-type PDEs. It completely eliminates the time dependency of the problem and the need for time discretization, yielding solutions numerical in space and semi-analytical in time. In LTBE solutions are obtained in the Laplace space, and are then inverted numerically to yield the soluticn in time. The Stehfest and the DeHoog formulations of LTBE, based on two different i'version algorithms, are investigated. Both formulations produce comparable, extremriy accurate solutions. The Stehfest formulation uses real values for the Laplace space parameter $\lambda$, combines linearly the results of a limited number of matrix solutions ( 6 to 8 ), does not increase computer storage, is simple to code, and requires significantly less execution time, but $y$ ields a solution at a single observation time $t$ for each set of $\lambda$ 's. The DeHoog formulation uses complex vislues for the $\lambda$ 's, needs more matrix inversions, and uses non-linear combinations of the solutions, but allows solutions at a range of times $t$ from a single set of $\lambda$ 's. Compared to the Stehfest LTBE, the DeHoog LTBE produces matrices 4 times as large, increases execution times per matrix inversion by at least a factor of 12 and the memory requirements by minimurn factor of 4 . The Stehfert LTBE seems to have a clear advantage, except in cases involving very steep functions of time.

\section{INTRODUCTION}

In diffusion-type equations, we seek an approximate solution to the PDE

$$
\nabla^{2} U\left(x_{\kappa}, t\right)=\frac{1}{k} \frac{\partial U\left(x_{\kappa}, t\right)}{\partial t} \quad x_{\kappa} \in \Omega
$$

with the boundary conditions

$$
\begin{gathered}
U\left(x_{\kappa}, t\right)=\bar{U}\left(x_{\kappa}, t\right), \quad x_{\kappa} \in \Gamma_{1}, \\
V\left(x_{\kappa}, t\right)=\frac{\partial U\left(x_{\kappa}, t\right)}{\partial n\left(x_{\kappa}\right)}=\bar{V}\left(x_{\kappa}, t\right), \quad x_{\kappa} \in \Gamma_{2},
\end{gathered}
$$

and initial conditions

$$
U\left(x_{\kappa}, t\right)=U_{0}\left(x_{\kappa}, t_{0}\right) .
$$


$\Omega$ is the solution domain. (a volume in three-dimensions and an area in two dimensions) of $U$, and $x_{\kappa}(\kappa \equiv 1,2,3)$ are the space coordinates. $\Gamma_{1}$ and $\Gamma_{2}$ are complementary segments of the exterior surface $\Gamma$ (an area in three-dimensions and a line in two dimensions), and $n$ is the unit outward vector normal to $\Gamma$. $U_{0}$ indicates known initial conditions, and $\bar{U}$ and $\bar{V}$ indicate known values of $U$ and $V$ on the boundaries. The term $k$ is assumed constant in time and space, and its interpretation depends on the physical problem under study. Parameter invariability does not limit the generality of equation (1). In homogeneous and anisotropic conditions are easily included by using subregions (e.g. Brebbia [1]) and stretched coordinates (e.g.liggett and Liu [2]).

Two traditional methods are used to address the problem of time dependency in equation (1). The first uses a coupled boundary element-finite difference approach in whish a finite difference approximation of $\frac{\partial U}{\partial t}$ is employed. This formulation, thoroughly investigated by Curan, Cross, and Lewis [3], needs small time steps to produce accurate results, and requires large computation times. The second formulation [4] accounts for time dependence by directly integrating over time a weighted residuals equation of the PDE in (1), and uses time inter of higher order) and a time-marching procedure. These two time-marching schemes (e.g. Wrobel and Brebbia [5]) were discussed in detail by Brebbia [1]. Application of the BEM method, and discretization of the resulting equations results in a system of linear equations of the type $\mathbf{A} \vec{Y}=\vec{R}$, where $\mathbf{A}$ is s fully ponplated $N \times N$ matrix, $\vec{Y}$ is the vector of unknown (U's or $V$ 's), and $\vec{R}$ is the known right-hand side of the discretized equations. The time dependence, the complexity of determination of the elements of the matrix A (compounded by the elaborate time interpolation and time-marching schemes), and the fact that $\mathbf{A}$ is a fully populated matrix (unable to benefit from the wealth of fast matrix solvers available for the solution of banded matrices) results in a laboricus and computer-time intensive numerical method which has limited the adoption of BEM for the simulation of transient diffusion-type problems.

The combination of Laplace transforms with the BEM for the solution of (1) has been attempted in the past, but was hampered by the lack of a reliable algorithrn for the inversion from the Laplace space into the original time space. Using BEM and the inversion method of Schapery [6], Rizzo and Shippy [7] solved the Laplace-transformed equation of heat conduction. This inversion method had several serious weaknesses which limited its application. It was essentially a curve-fitting process, and as such presupposed some knowledge of the expected solution. The Laplace space transform parameter $\lambda$ was arbitrarily chosen, and a poor choice resulted in unstable solutions or insufficient definition of the curve and reduced accuracy (e.g. Liggett and Liu [8]). The curve fitting scheme required the determination of $N$ coefficients obtained from the computertime consuming solution of a system of simultaneous equations, and was impractical and time-consurning if the boundaries had a complex tine history (Lachat and Combescure $[9])$.

The Laplace Transform Boundary Element (LTBE) numerical method, is a recently developed numerical method introduced by Moridis and Reddell [10]. It belongs to it family of Laplace transform-based numerical methods introduced by Moridis and Reddell $\{11,12,13,14]$, and does not suffer from the limitations of the Schapery [0] niversion scheme. The method eliminates protlems with stability and accuracy posed by the traditional treatment of the time clerivative, renders the time interpolation and time marching schemes irrelevant, and allows an unlimited $\Delta t$ size without any loss of accuracy 
or stability because time is no longer considered. It consists of 4 steps: (1) A Laplace transform is performed on the PDE, (2) the transformed PDE is approximated using the Boundary Elenent Method (BEM), (3) the resulting system of simultaneous equations is solved and the transformed vector of unknowns is determined in the Laplace space, and (4) the solution vector obtained in step 3 is inverted numerically to yield the solution in time. In this paper two alternative formulations of the LTBE, based on different numerical inversion schemes, are presented and compared.

\section{THE ITBE NUMERICAL METHOD}

In this section the mathematical basis of LTBE is presented and the four steps involved in its application are described.

\section{Step 1: The Laplace Transform of the PDE}

Because of the properties of the Laplace transform, equation (1) bacomes in the Laplace transform space

$$
\nabla^{2} \Psi\left(x_{\kappa}, \lambda\right)=\frac{1}{k}\left[\lambda \Psi\left(x_{\kappa}, \lambda\right)-U_{0}\left(x_{\kappa}, t_{0}\right)\right]
$$

with the boundary conditions

$$
\begin{gathered}
\Psi\left(x_{\kappa}, \lambda\right)=\bar{\Psi}\left(x_{\kappa}, \lambda\right), \quad x_{\kappa} \in \Gamma_{1}, \\
\Theta\left(x_{\kappa}, \lambda\right)=\frac{\partial \Psi\left(x_{\kappa}, \lambda\right)}{\partial n\left(x_{\kappa}\right)}=\bar{\Theta}\left(x_{\kappa}, \lambda\right), \quad x_{\kappa} \in \Gamma_{2},
\end{gathered}
$$

where $\lambda$ is the Laplace space transform parameter,

$$
\Psi\left(x_{\kappa}, \lambda\right)=\mathcal{L}\left\{U\left(x_{k}, t\right)\right\}, \quad \Theta\left(x_{\kappa}, \lambda\right)=\mathcal{L}\left\{V\left(x_{\kappa}, t\right)\right\},
$$

and $\mathcal{L}\{\}$ denotes the Laplace transform of the quantity in brackets. If sources or sinks of strength $Q$ are included, then the term $\ddot{Q}\left(x_{\kappa}, \lambda\right)=\mathcal{L}\left\{Q\left(x_{*}, t\right)\right\}$ is added to the right-hand side of equation (5).

Step 2: The BEM in the Laplace Space

For an appropriate fundamental solution $\Psi^{*}$, the minimization of the weighted residuals using the BEM at all interior points $\xi$ yields the statement

$$
\begin{aligned}
\Psi(\xi, \lambda)= & k \int_{\Gamma} \Theta\left(x_{\kappa}, \lambda\right) \Psi^{*}\left(\xi, x_{\kappa}, \lambda\right) d \Gamma\left(x_{\kappa}\right)-k \int_{\Gamma} \Psi\left(x_{\kappa}, \lambda\right) \Theta^{*}\left(\xi, x_{\kappa}, \lambda\right) d \Gamma\left(x_{\kappa}\right)+ \\
& \int_{\Omega} U_{0}\left(x_{\kappa}, t_{0}\right) \Psi^{*}\left(\xi, x_{\kappa}, \lambda\right) d \Omega\left(x_{\kappa}\right)-\int_{\Omega} \tilde{Q}\left(x_{\kappa}, \lambda\right) \Psi^{*}\left(\xi, x_{\kappa}, \lambda\right) d \Omega\left(x_{\kappa}\right),
\end{aligned}
$$

where $\Theta^{*}\left(\xi, x_{\kappa}, \lambda\right)=\partial \Psi^{*}\left(\xi, x_{\kappa}, \lambda\right) / \partial n\left(x_{\kappa}\right)$. The fundamental solutions $\Psi^{*}$ for the twoand the three-dimensional problem are given by Brebbia, Telles, and Wrrbel [4] and Greenberg [15], and are respectively

$$
\Psi^{*}=\frac{1}{2 \pi k} \cdot K_{0}\{r \sqrt{T / k}\} \text { and } \Psi^{*}=\frac{(k \lambda)^{1 / 4}}{r^{1 / 2} \cdot(2 \pi k)^{3 / 2}} \cdot K_{1 / 2}\{r \sqrt{\lambda / k}\}
$$

where $K_{m}$ is the motified Bessel function of the second kind and of order $m$. The $\Psi^{*}$ of the three-dimensional problem has a singularity as $r \rightarrow 0$, with a limiting form of 
$\Psi^{*}=1 /(4 \pi k r)$ (Abramowitz and Stegun [16]). The $\Psi^{*}$ of the two-dimensional problem is singular as $r \sqrt{\lambda / k} \rightarrow 0$ [14], with a limiting form

$$
\lim \Psi^{*}=\frac{1}{2 \pi k} \ln \frac{1}{r}-\frac{1}{4 \pi k} \ln \frac{\lambda}{k} \text { for } r \sqrt{\lambda / k} \rightarrow 0 .
$$

For the point $\xi$ approaching $\Gamma$, the equation to be solved is

$$
\begin{aligned}
C(\xi) \Psi(\xi, \lambda)= & k \int_{\Gamma} \Theta\left(x_{\kappa}, \lambda\right) \Psi^{*}\left(\xi, x_{\kappa}, \lambda\right) d \Gamma\left(x_{\kappa}\right)-k \int_{\Gamma} \Psi\left(x_{\kappa}, \lambda\right) \Theta^{*}\left(\xi, x_{\kappa}, \lambda\right) d \Gamma\left(x_{\kappa}\right)+ \\
& \int_{\Omega} U_{0}\left(x_{\kappa}, t_{0}\right) \Psi^{*}\left(\xi, x_{\kappa}, \lambda\right) d \Omega\left(x_{\kappa}\right)-\int_{\Omega} \tilde{Q}\left(x_{\kappa}, \lambda\right) \Psi^{*}\left(\xi, x_{\kappa}, \lambda\right) d \Omega\left(x_{\kappa}\right),
\end{aligned}
$$

in which $C(\xi)$ is a coefficient and $\theta^{*}$ is discontinuous as $\xi \rightarrow \Gamma$. Discretization of equation (12) on the boundary $\Gamma^{\prime}$ yields the relationship beiween the node $i$ at which the fundamental solution is applied and all the elements of the boundary, and has the form.

$$
\begin{aligned}
C_{i} \Psi_{i}+ & \overbrace{\sum_{j=1}^{N} k \int_{j} \Psi \Theta^{*} d \Gamma}^{\Delta_{1 i}} \overbrace{\sum_{j=1}^{N} k \int_{j} \Psi^{*} \Theta d \Gamma-}^{\Delta_{2 i}} \\
& \underbrace{\sum_{N_{e}}\left[\sum_{m=1}^{M} w_{m}\left(U_{0} \Psi^{*}\right)_{m}\right] A_{c}+}_{B_{i}}+\underbrace{\sum_{N_{a}}\left[\sum_{m=1}^{M} w_{m}\left(\tilde{Q} \Psi^{*}\right)_{m}\right]}_{T_{i}} A_{c}=0,
\end{aligned}
$$

where $\Gamma_{j}$ is the length of the element $j, w_{m}$ are the integration weights, and $N_{e}$ is the number of cells (of area $A_{e}$ ) into which $\Omega$ is subdivided. The quantities $\left(U_{0} \Psi^{*}\right)_{m}$ and $\left(\tilde{Q} \Psi^{*}\right)_{m}$ must be evaluated at the $M$ integration points. The quantity $B_{i}$ represents a domain integral resulting from the imposition of specific initial conditions. The quantity $T_{i}$ represents the domain integral of sousce and sink singularities usually appearing in the interior of $\Omega$, and allows an easy accounting for the effects of sources and sinks if a better definition of $U$ and $V$ in their vicinity is not needed. $B_{i}$ and $T_{i}$ do not introduce any more unknowns since both $U_{0}$ and $Q$ are prescribed. If $U_{0}$ satisfies Laplace's equation or is harmonic in $\Omega$, the domain integral of $U_{0}$ can be transformed into equivalent boundary integrals [1], and integration over the $N_{e}$ cells of $\Omega$ to obtain $B_{i}$ is avoided.

If constant boundary elements are employed, $C_{i} \equiv \frac{1}{2}$, and $\Psi$ and $\Theta$ are constant within each eiement. Then $\Delta_{1 i}$ and $\Delta_{2 i}$ become

$$
\Delta_{1 i}=\sum_{j=1}^{N} \ddot{H}_{i, j} \Psi_{j}, \quad \Delta_{2 i}=\sum_{j=1}^{N} G_{i, j} \Theta_{j}, \text { where } \hat{H}=k \int_{\Gamma} \Theta^{*} d \Gamma \text { and } G=k \int_{\Gamma} \Psi^{*} d \Gamma .
$$

Substituting in equation (13), we obtain

$$
\sum_{j=1}^{N} H_{i, j} \Psi_{j}-\sum_{j=1}^{N} G_{i, j} \Theta_{j}-B_{i}+T_{i}=0
$$

where $H_{i j}=\hat{H}_{i, j}$ if $i \neq j$ and $H_{i j}=\ddot{H}_{i, j}+\frac{1}{2}$ if $i=j$. Caution must be used in the calculation of $H_{i j}$ and $G_{i, j}$ in the vicinity of singularities (usually as $r \rightarrow 0$ ), in which case 
higher order integration schemes need to be used, e.g. Brebbia, Telles, and Wrobel [4], Ligget and Liu [2]. Collecting and rearranging terms, we arrive at the matrix equation

$$
\mathbf{H} \vec{\Psi}-\mathbf{G} \vec{\Theta}=\vec{R}, \quad \vec{R}=\vec{B}-\vec{T} .
$$

The total number of unknown $\Psi$ 's and $\Theta$ 's in equation (16) is $N$. Separating and reordering knowns and unknowns yields the final BEM system of simultaneous linear equations described by the matrix equation

$$
\mathbf{A} \vec{Y}=\vec{R},
$$

in which $\vec{Y}$ is the vector of unknown $\Psi$ 's and $\Theta$ 's, $\vec{R}$ is the vector of the known righthand side, and $\mathrm{A}$ is the fully populated $N \times N$ coefficient matrix. The use of constant elements in our analysis does not affect the generalit: of the LTBE method. If linear or higher order elements are used, the resulting final equations are invariably of the type of equations (15), (16) and (17), and all subsequent procedures apply.

\section{Step 3: The Solution in the Laplace Space}

The computation of $\mathbf{A}$ and $\vec{R}$ necessitates values for the $\lambda$ parameter of the Laplace space. These are provided by the two schernes investigated in this paper: the Stehfest algorithm (Stehfest $[17,18]$ ), and the DeHoog method (DeHoog, Kinght, and Stokes [19]). For a desired observation time $t$, the $\lambda$ in the Stehfest $[17,18]$ algorithm is real and given by

$$
\lambda_{\nu}=\frac{\ln 2}{t} \cdot \nu, \quad \nu=1, \ldots, N_{S}
$$

where $N_{S}$ is the number of summation terms in the algorithm ard is an even number. Optimum values for $N_{S}$ are discussed in a following section. In the DeHoog method [19], $\lambda$ is a complex number given by Sudicky [20] and Crump [21] as

$$
\lambda_{\nu}=\lambda_{0}+\frac{\nu \pi \iota}{T}, \quad \lambda_{0}=\mu-\frac{\ln \left(E_{R}\right)}{2 T}, \quad \nu=1, \ldots, N_{H}
$$

where $2 T$ is the period of the Fourier series approximating the inverse function in the interval $[0,2 T], \iota=\sqrt{-1}$, and $N_{H}=2 M+1$ is an odd number. A discisssion of the terms $\mu$ and $E_{R}$ can be found in Sudicky [20]. As is later discussed, excellent results are obtained when $\mu=0,10^{-10} \leq E_{R} \leq 10^{-8}$, and $0.9 t_{\max } \leq T \leq 1.1 t_{\max }$, where $t_{\max }$ is the maximurn simulation time.

The solution of (17) returns a set of $N_{S}$ or $N_{H}$ vectors of the unknown $\vec{Y}$ 's as

$$
\vec{Y}_{\nu}=\vec{Y}\left(\lambda_{\nu}\right)=\left[\mathrm{A}\left(\lambda_{\nu}\right)\right]^{-1} \vec{R}\left(\lambda_{\nu}\right), \quad \nu=1, \ldots, N_{S} \text { or } N_{H}
$$

To obtain a solution at a time $t$, all vectors $\vec{Y}_{\nu}, \nu=1, \ldots, N_{S}$ or $N_{H}$ are needed, i.e. the system of simultaneous equations has to be solved $N_{S}$ or $N_{K I}$ times. Once $\vec{Y}_{\nu}$ is known, the value of $\left[\Psi\left(\lambda_{\nu}\right)\right]_{i}$ at any interior point $i$ can be determined from the discretized integral relationship between $i$ and the bundary values of $\Psi\left(\lambda_{\nu}\right)$ and $\Theta\left(\lambda_{\nu}\right)$ as

$$
\left[\Psi\left(\lambda_{\nu}\right)\right]_{i}=\sum_{j=1}^{N} G_{i, j}\left[\Theta\left(\lambda_{\nu}\right)\right]_{j}-\sum_{j=1}^{N} \hat{H}_{i, j}\left[\Psi\left(\lambda_{\nu}\right)\right]_{j}+B_{i}-T_{i}
$$


Step 4: The Numerical Inversion of the Laplace Solution

The unknown $U$ 's at any boundary or interior point $i$ and at any time $t$ are obtained by numerically inverting the Laplace space solutions $\Psi_{i}\left(\lambda_{\nu}\right)$. When using the Stehfest [17, 18 ] algorithm, the procedure is described by the following equations:

$$
[U(t)]_{i}=\frac{\ln 2}{t} \sum_{v=1}^{N_{s}} W_{\nu} \cdot\left[\Psi\left(\lambda_{v}\right)\right]_{i},
$$

where

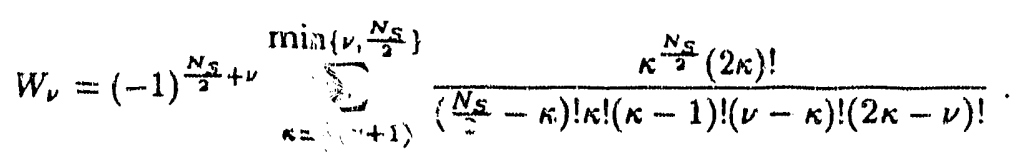

Although the accuracy of the meth $t$ is theoretically expected to improve with increasing $N_{S}$, Stehfesi [17] showed that wii. increasing $N_{\mathcal{S}}$ the number of correct significant figures inreases linearly at first and then, due to roundoff errors, decreases linearly. $\mathrm{He}$ determined that the optimum $N_{S}$ was 10 for single precision variables (8 significant figures) and 18 for double precision variables (16 significant figures). However, Moridis and Reddell [10] reported that LTBE seems to be insersitive to $N_{S}$ for $6 \leq N_{S} \leq 20$.

The inversion of the Laplace space solution obtained with the DeHoog [19] method is far more complicated. The solution at a time $t$ is given by

$$
[U(t)]_{i}=\frac{1}{T} \exp \left(\lambda_{0} t\right) \operatorname{Re}\left\{\frac{A_{2 M}}{B_{2 M}}\right\}
$$

where $A_{-1}=0, A_{0}=d_{0}, B_{-1}=B_{0}=1$,

$$
\begin{gathered}
A_{n}=A_{n-1}+d_{n} z A_{n-2}, \quad B_{n}=B_{n-1}+d_{n} z B_{n-2}, \quad n=1, \ldots, 2 M, \\
d_{0}=a_{0}, \quad d_{2 m-1}=-q_{m}^{(0)}, \quad d_{2 m}=-e_{m}^{(0)}, \quad m=1, \ldots, M, \\
\ell=1, \ldots, M, \quad e_{\ell}^{(\kappa)}=q_{\ell}^{(\kappa+1)}-q_{\ell}^{(\kappa)}+e_{\ell-1}^{(\kappa+1)}, \quad \kappa=0, \ldots, 2 M-2 \ell \\
\text { for } \ell=2, \ldots, M, \quad q_{\ell}^{(\kappa)}=q_{\ell-1}^{(\kappa+1)} e_{\ell-1}^{(\kappa+1)} / e_{\ell-1}^{(\kappa)}, \quad \kappa=0, \ldots, 2 M-2 \ell-1, \\
e_{0}^{(\kappa)}=0 \text { for } \kappa=0, \ldots, 2 M \quad \text { and } \quad q_{1}^{(\kappa)}=a_{\kappa+1} / a_{\kappa} \text { for } \kappa=0, \ldots, 2 M-1,
\end{gathered}
$$

and

$$
a_{0}=\frac{1}{2} \Psi\left(\lambda_{0}\right), \quad a_{\kappa}=\Psi\left(\lambda_{\kappa}\right) \text { and } z=\exp \left(\iota \pi t / T^{\prime} .\right.
$$

A further acceleration is obtained if on the last evaluation of the recurrence relations $d_{2 N} z$ is repaced by $R_{2 M}(z)$,

$$
R_{2 M}(z)=-h_{2 M}\left[1-\sqrt{\left(1+d_{2 M} z / h_{2 M}\right)}\right], \quad h_{2 M}=\frac{1}{2}\left[1+z\left(d_{2 M-1}-d_{2 M}\right)\right],
$$

giving

$$
\hat{A}_{2 M}=A_{2 M-1}+R_{2 M} A_{2 M-2}, \quad \hat{B}_{2 M}=B_{2 M-1}+R_{2 M} B_{2 M-2},
$$

in which case the accelerated solution at a time $t$ is given by

$$
[U(t)]_{i}=\frac{1}{T} \exp \left(\lambda_{0} t\right) \operatorname{Re}\left\{\frac{\hat{A}_{2 M}}{\hat{B}_{2 M}}\right\} .
$$


As will be shown in a subsequent section, the minimum $M$ for an acceptable accuracy is 5 , resulting in a $N_{H}=11$, which indicates that equation (17) has to be solved a minimum of 11 times. For an accuracy comparable to that of the Stehfest method $M \geq 6$ and $N_{H} \geq 13$. The unique advantage of the DeHoog formulation is that a whole range of solutions at times $t$ in the range $[0, T]$ can be obtained from a single set of solutions $\Psi$, i.e. equation (17) needs not be solved for each $t$ of interest.

The solution in the Laplace space eliminates stability and accuracy problems caused by the treatment of the time derivative in standard BEM simulators, thus allowing an unlimited time-step size. The truncation error of the method is limited to that caused by the space discretization because time is not discretized, and provides a solution inherently more accurate than the standard BEM method for the same grid system. The ability to use an unlimited time-step size bounds the accumulation of roundoff error by an upper limit defined as the roundoff error accumulated after the $N_{S}$ or $N_{H}$ solutions. Thus, LTBE offers a stable, non-increasing roundoff error irrespective of the time of observation $t_{o b}$, because calculations are performed at one time only by letting $\Delta t=t_{\text {obs }}$. Calculations in the standard BEM method have to be performed at all the intermediate times of the discretized time domain, continuously accumulating roundoff error in the process.

\section{THEORETICAL COMPARISONS OF 'THE TWO LTBE FORMULATIONS}

\section{Computer Memory Requirernents}

This analysis excludes the memory requirement for property description and domain discretization (which are identical in both formulations), and focuses on the mernory needed to obtain the solutions of equations (22) and (24) or (32). In the Stehfest LTBE, the memory requirements are limited to the storage needed for (a) the fully populated matrix in equation (17), (b) the vector of the initial conditions, (c) the solution vector $\vec{Y}_{\nu}$ in the Laplace space, (d) the vector of the interior points in equation (21), and (e) an intermediate vector, which, because of the simple additive algorithm of equation (22), is also used to store the solution at time $t$. If $N$ is the number of boundary nodes and $N_{i}$ the number of interior points at which a solution is required, then the total memory requirement (number of double precision words) for the Stehfest LTBE is

$$
M_{S t}=\overbrace{N(N+1)}^{(a)}+\overbrace{N}^{(b)}+\overbrace{N}^{(c)}+\overbrace{N_{i}}^{(d)}+\overbrace{N_{i}}^{(e)}=N^{2}+3 N+2 N_{i} .
$$

The situation is drastically different for the DeHoog LTBE. Because $\lambda_{\nu}(\nu>0)$ is a complex number, all the pertinent matrices and vectors are complex. Use of complex arithmetic on a computer for the solution of the matrix equation (17) may be convenient, but this approach is extremely inefficient and slow. A much better approach is to split each node equation in two: the first equation is the equation of the real parts, and the second is the equation of the imaginary parts. Then there is no need to use complex arithmetic for the solution of equation (17), the vectors $\vec{Y}$ and $\vec{R}$ become real vectors of length $2 N$ each, and matrix $\mathbf{A}$ is a fully populated $2 N \times 2 N$ real matrix. In the inversion of the Laplace space solution using the DeHoog method all the intermediate vectors $\vec{A}, \vec{B}, \vec{d}, \vec{q}, \vec{e}$ must be stored, and complex arithmetic must be used. Using the same nomenclature as in equation (33), the storage requirement (in double precision words) is

$$
\begin{aligned}
M_{D H} & =\overbrace{2 N(2 N+1)}^{(a)}+\overbrace{2 N}^{(b)}+\overbrace{2 N}^{(c)}+\overbrace{(2 M+1) N_{i}}^{(d)}+\overbrace{2 N_{i}\left(2 M^{2}+11 M+7\right)}^{(f)} \\
& =4 N^{2}+6 N+N_{i}\left(4 M^{2}+24 M+17\right),
\end{aligned}
$$


where $N_{H}=2 M+1$ (equations (24) through (32)), and (f) indicates the memory requirement for the intermediate yectors and corresponds to an optimized memory management. The strong dependence of $M_{D H}$ on $M$ and $N_{i}$ is obvious. Figure 1 shows the memory requirement ratio $R_{M R}=M_{D H} / M_{S t}$ of the two formulations for a number of cases for $M=5$ (the absolute minimurn). It is evident that (1) the DeHoog LTBE requires at least 4 times the storage of the Stehfest LTBE, (2) $R_{M R}$ increases with the number of internal nodes of interest $N_{i}$, and (3) $R_{M R}$ is significantly higher for $N<100$ (usually the case in most LTBE simulations), demonstrating the advantage of the Stehfest LTBE. This advantage becomes more pronounced with increasing $M$.

\section{Computational Effort}

The matrix in equation (17) is a ully populated matrix, and as such it is unable to benefit from the wealth of fast matrix solvers available for the solution of banded matrices. The solution of (17) is by far the most, computer time-intensive process in LTBE. This analysis de ermines the work of a Gauss elimination solver, and accounts for all the divisions, multiplications, additions, subtractions, and register access operations (i.e. transfers between registers and the main memory of the computer) involved in a single matrix inversion. For the Stehfest LTBE scheme, the number of divisions is

$$
L_{S t}^{(1 / x)}=\sum_{\ell=1}^{N} 1=N
$$

the number of multiplications is

$$
L_{S t}^{(*)}=\sum_{\ell=1}^{N}\left(\ell^{2}+\ell-1\right)=\frac{1}{6} N(N+1)(2 N+1)+\frac{1}{2} N(N+1)-N,
$$

the number of additions and subtractions is

$$
L_{S t}^{( \pm)}=\sum_{\ell=1}^{N}(\ell-1)(\ell+1)=\frac{1}{6} N(N+1)(2 N+1)-N,
$$

and the number of RAO's is

$$
L_{S t}^{(R A)}=\sum_{\ell=1}^{N}[\ell(4 \ell+1)+2]=\frac{2}{3} N(N+1)(2 N+1)+\frac{1}{2} N(N+1)+2 N .
$$

In the Defloog formulation, for $\lambda=\lambda_{0}$ the equation to be solved (and the corresponding computational effort) are practically the same as in the case of the Stehfest version because $\lambda_{0}$ is a real number and all the constituents of equation (17) are real. For $\lambda_{\kappa}$ with $\kappa \geq 1$, the equation splitting technique discussed in the previous subsection results in a fully populated $2 N \times 2 N$ real matrix $\mathbf{A}$. Then the work for divisions, multiplications, additions/subtractions, and RAO's are obtained from equations (35) through (38) by replacing $N$ by $2 N$ in the summations.

The work to obtain $\vec{Y}$ is then computed as

$$
W_{T}=C_{C}^{(1 / x)} \cdot L_{T}^{(1 / x)}+C_{C}^{(*)} \cdot L_{T}^{(*)}+C_{C}^{( \pm)} \cdot L_{T}^{( \pm)}+C_{C}^{(R A)} \cdot L_{T}^{(R A)} ; \quad T \equiv S t, D h,
$$

where $C_{C}^{(1 / x)}, C_{C}^{(*)}, C_{C}^{( \pm)}$, and $C_{C}^{(R A)}$ are the computer clock cycles per division, multiplication, addition or subtraction, and RAO. The numbers of clock cycles per 
operation for a number of different computers are presented in Table 1. Figure 2 shows the work ratio $R_{W}=W_{D H} / W_{S t}$ when $M=5$ for a single matrix solution and for a variety of computing platforms. The larger the value of $R_{W}$, the larger the execution time required by the DeHoog LTBE and the less attractive the method compared to the Stehfest version. It can be seen that (1) a single matrix solution in the DeHoog LTBE method requires at least 6 times the work (and the execution time) of the Stehfest LTBE, (2) $R_{W^{\prime}}$ rapidly reaches the asymptotic value of $(2 N / N)^{3}=8$, and (3) machine specificity is not an important factor.

Table 1. Clock Cycles per Double Precision Operation for Various Computers

\begin{tabular}{|c|c|c|c|c|c|c|}
\hline COMPUTER & $a+1-b$ & $a^{*} b$ & $1 / b$ & $a *(1 / b)$ & Register Access Operations & RAO \\
\hline & & & & & Memory to Cache-1st element & 22 \\
\hline \multirow[t]{3}{*}{ IBM 3090} & 3 & 5 & 30 & 30 & Memory to Cache-Next 15 elements & 15 \\
\hline & & & & & Cache to Register -16 elements & 16 \\
\hline & & & & & TOTAL for 16 array elements & 53 \\
\hline Prime 2275 & 1 & 13 & 34 & 47 & & 2 \\
\hline Cray-YMP & 6 & 7 & 14 & 21 & Memory to Register - Scalar & 17 \\
\hline
\end{tabular}

\section{EXPERIMENTAL COMPARISONS OF THE TWO LTBE FORMUI ATIONS}

The two versions of the LTBE numerical method were tested in four fiffusion-type problems of groundwater flow which represented increasing levels of complexity The fact that the examples focus exclusively on groundwater problems does not in any way limit the generality of the method, and an identical approach can be employed within the same class of PDE's. In the first test problem, the LTBE solutions were verified through comparison to the existing analytical solution. No analytical solution exists for the remaining test cases. In all four cases the results obtained from the implementation of LTBE were tested against results obtained from a standard BEM simulator for the same space discretization. Based on the previous work of Moridis and Reddell [10], a $N_{S}=6$ was used in all the Stehfest LTBE simulation. The value of $M=6\left(N_{H}=13\right)$, which was shown to result in an accuracy compdrable to that of the Stehfest LTBE, was used in the Delloog LTBE simulations.The simultaneous equations of the LTBE and BEM methods were solved by a Gauss elimination. Linear space interpolation functions were used in all cases. In the BEM simulator a constant time interpolation was employed. Double precision variables with 20 significant figures were used in all simulations.

\section{Verification \& Test Case 1}

Test case 1 represents the one-dimensional radial flow problem towards a well of radius $r_{w} \rightarrow 0$ in a homogeneous circular aquifer with infinite boundaries. The aquifer had a transmissivity $T=1000 \mathrm{~m}^{2} /$ day, a storage coefficient $S=0.0001$, and water was pumped at a rate of $Q=1000 \mathrm{~m}^{3} /$ day. A uniform thickness of $50 \mathrm{~m}$ was assumed, and the initial aquifer pressure was taken as $p_{0}=6 \times 10^{5} P_{a}$. A single observation was made at $t_{o b s}=10$ days. A variable $\Delta t$ was used for all test cases in the BEM simulator, given by the recursive formula $\Delta t_{\ell}=\min \left\{1.5 \times \Delta t_{\ell-1}, \Delta t_{\max }\right\}$, with $\Delta t_{\max }=8.64 \times 10^{4} \mathrm{sec}=1 \mathrm{day}$. The number of time-steps was therefore a function of the original time-step $\Delta t_{0}$.

The analytical solution was given by Theis [22]. Varying $\Delta i_{0}$ in the BEM simulator, the number of required time-steps to reach $t_{o b s}$ was $1,2,3,4,0$, and 18 . The drawdown 
results for both the analytical and the numerical solutions, as well as the difference between the Theis solution and the numerical solutions, are presented in Figures 3(a) and $3(b)$ respectively. The Theis solution and the two LTBE solutions (obtained using a total of $N_{i}=60$ internal points) practically coincided, having a maximum difference of $1.0 \times 10^{-3} \mathrm{~m}$ at $r=0.01 \mathrm{~m}$ (where the maximum drawdown of $2.3487 \mathrm{~m}$ occurs). As expected, the BEM solution tended towards the LTBE and the Theis solutions with an increasing number of time-steps (smaller $\Delta t$ 's). Both LTBE variants give extremely accurate results. The Stehfest LTBE solution seems to be slightly more accurate, but the difference between the two LTBE versions is too small to be of any practical significance.

\section{Test Case 2}

The second test problem involved flow towards a single well in a twudimensional axisymmetric cylindrical $(r, z)$ system. The geometry, boundaries, and properties of the simulated aquifer (i.e. the permeabilities $k_{r}, k_{z}$ in $(r, z)$ and the porosities $\phi$ ), as well as other general information, are shown in Figure 4 , and describe an extremely anisotropic and inhomogeneous layered system. Since no analytical solution is available, a comparison was made between the LTBE and the BEM solutions. The initial pressure and the boundary pressure at the constant head boundary were $p_{0}=6.0 \times 10^{5} P a$. All other properties were as in the Test Case 1.

The formulation of the equations followed the procedure described by Liggett and Liu [2]. The domain was subdivided into 5 subregions, and stretched coordinates were used to describe the anisotropy of the problem. For the LTBL and the BEM sirnulations the internal and external boundaries were divided in 160 unequally-sized elements, resulting in a total of $N=154$ nodes. A single observation was made at $t_{o b s}=12 \mathrm{hrs}$. Varying $\Delta t_{0}$ in the BEM simulator, the number of required time-steps to reach $t_{o b s}$ was $1,2,5$, and 28 . Comparisons of the drawdown estimated from the LTBE and the BEM solutions were made along the $z$ axis at $r=0.15 m$ using a total of $N_{i}=25$ internal points. The comparison appears in Figure 5 , in which the sarne pattern as in the Test Case 1 is evident. The accuracy of the LTBE solutions was indicated by the fact that the BEM solution for an increasing number of $\Delta t$ 's (corresponding to a decreasing $\Delta t$ size, smaller truncation errors, and more accurate solutions) tended to the LTBE solutions. The power of the LTBE methods is demonstrated in Figure 5 by its ability to capture in detail the significant variations in drawdown due to the presence of wells and zones of drastically different permeability. The standard BEM solution showed significant, deviations and insufficient accuracy caused by the averaging effect of the treatment of the time derivative for larger $\Delta t$ 's, deviations which decreased with smaller $\Delta t$ 's. The two LTBE solutions are extremely close. As is shown below (Figure 9), the Stehfest ve!sion is more accurate, but the difference is negligible for any practical purposes.

\section{Test Case 3}

The third test problem was identical to the problern in Test Case 2 in properties, initial and boundary conditions, and space discretization. The different features in 'Test Case 3 are: (1) time-dependent, well rates, obeying the linear relationship $Q=$ $Q_{0}\left(1-3.8580246 \times 10^{-7} t\right)$, where $Q_{0}$ is the initial rate (same as in Test Case 2), and $t$ is in seconds, and (2) tinue-variable boundary conditions (at the position of the timeinvariant boundary in Test Case 2) obeying the relationship $p_{b}(t)=p_{b}(0)+0.23148148 t$, where $p_{b}(t)$ is the time variable boundary pressure. A single observation was made at $t_{\text {obs }}=10$ days. In the BEM simulation equal time step sizes were used, resulting in 1 , $10,20,40,120$, and $240 \angle i$ 's.

Comparisons of the potential (head) estimated from the LTBE and the BEM 
solutions were made along the $z$ axis at $r=015 \mathrm{~m}$ using the same $N_{i}=25$ internal points. The results of the comparison appear in Figure 6, in which the same pattern observed in all previous test cases is obvious: the two LTBE versions produced accurate solutions, a fact indicated by the realization that the BEM solution for a decreasing $\Delta t$ size tended to the LTBE solutions. Compared to the BEM, the LTBE methods are better equiped to accurately describe the effects of the presence of wells and zones of significantly different permeability. These are identified by the existence of peaks and sharp variations in potential, variations which decrease in rnagnitude with a decreasing $\Delta t$ size in the BEM solution. Both the Stehiest and the DeHoog LTBE versions produce extremely accurate, virtually indistinguishable results; the Stehfest version has a slight accuracy advantage, but for practical purposes the two versions produce identical solutions.

\section{Test Case 4}

Test case 4 was an anisotropic and inhomogeneous three-dimensional cartesian $(x, y, z)$ system of flow to two wells. The initial and boundary conditions, geometry, boundaries, and properties of the aquifer, as well as the location and pumping rates of the two wells, are shown in Figure 7. The aquifer had a time-variable boundary at $x=8,000 \mathrm{~m}$. No analytical solution was possible for this problem. Using the procedure of Liggett and Liu's [2], the domain was subdivided into 3 subregions, and stretched coordinates were used to describe the heterogeneity of the problem.

The boundary of the solution domain was discretized into 772 unequally-sized triangular (linear) elements, with $N=364$. The interior domain was subdivided into 800 tetrahedral elements for the computation of $B_{i}$ and $T_{i}$. A single observation was made at $t_{o b_{s}}=10$ days. The LTBE methods were evaluated by comparing the golutions of the potential (head) distribution along the $x$ axis at $y=1050 \mathrm{~m}, z=17.5 \mathrm{~m}$ and passing through the well at $(x, y, z)=2000 \mathrm{~m}, 1050 \mathrm{~m}, 17.5 \mathrm{~m})$. The potential distribution is shown in Figure 8. The pattern observed in all previous test cases is present here. The power of LTBE and the inability of BEM - caused by the averaging effect of the treatment of the time derivetive for larger $\Delta t$ 's - to accurately describe the effects of wells and timevariable boundaries are reflected in the difference between the solutions. The location of the well can be identified by the existence of a 'valley' in Figure 8. With increasingly fine time discretization, the BEM solution approaches the two ITBE solutions. Both LTBE versions yield very accurate solutions which are very close to each other. The Stehfest LTBE method is more accurate, but not significantly so.

\section{Mass Balance Error Considerations}

A very important measure of (a) the validity and accuracy of the LTBE methods and (b) their relative performance was provided by the determination of the mass balance error $E_{B}$. Figure 9 shows $E_{B}$ for the Stehfest and the DeHoog LTBE methods, as well as the dependence of $E_{B}$ on $N_{S}$ and $N_{H}$. The following conclusions can be drawn: (1) the Stehfest LTBE version is consistently more accurate (and computationally more efficient) for a smaller number of matrix solutions $N_{S},(2)$ as a mass balance error of $10^{-3}$ $-10^{-2}$ is unacceptably high for most groundwater applications, the minimum acceptable number of matrix solutions $N_{H}$ in the DeHoog LTBE is $11(M=5),(3)$ an accuracy comparable to that of the Stehfest LTBE version is attained for $N_{H} \geq 13(M \geq 6)$, (4) the performance of the Delloog LTBE seems to be far more adversely affected by the complexity of the problem, (5) the performance of the Stehfest LTBE scheme improves initially (but not monotonically) with an increasing $N_{S}$, and seems to be practically insensitive to the value of $N_{S}$ for $6 \leq N_{S} \leq 16$, as the arithmetic differences of the solutions are at or beyond the 4th or 5 th decimal place, and (6) the performance of the DeHoog LTBE method improves monotonically with an increasing $N_{H}$. 
These last two observations confirm the theoretical background of the two inversion methods. The Stehfest algorithm is based on coefficients computed as ratios of factorials (equation (23)), which may be theoretically correct but may introduce computational errors because the rapidly increasing magnitudes and the resulting roundoff error may overwithelm the machine accuracy after a certain $N_{S}$ (which seems to be problem-specific). This can be evidenced by the existence of a minimum in the mass balance error curve. On the other hand, the DeHoog method is based on the Pade' approximant of an infinite sum, and its accuracy beeps increasing with the number of terms involved ( $M$ or $N_{H}$ ) until the rnachine accuracy is reached. This is indicated by the monotonically declining mass balance error in the Delloog LTBE curve, and is an advantage of the rnethod in the inversion of very steep functions of time such as step functions and spikes. This seems to be the only case when the Stehfest algorithm is at a disadvantage, as it tends to smear these steep fronts. Stehfest [17] identified this weakness, and cautioned against using the algorithm in the inversion of extremely steep surfaces. Increasing $N_{S}$ to 20 or 22 improves its performance, but small cocillations and some smearing persist. However, such steep time functions may be encountered only if well rates or boundary conditions are described by step functions or pulses. The Delloog method is ideally suited to the inversion of sum st.ep time functions, and retuins extrernely accurate solutions with an $M \geq 10\left(N_{H} \geq 21\right)$. Unless the simulated processes cannot be described otherwise (e.g. by interrupting and restarting the simulation with a different well race instead of using a step function), the excessive storage and (xecution time requirements make the use of the DeHoog LTBE methoi prohibitive.

Having determined the minimum $M$ for an acceptable level accuracy of the DeHoog LTBE scherne, the ratio of the total work for the two LTBE versions can be determined as $R_{T W}=W_{1 D, r} / W_{S t, r}$, where the terms $W_{D H_{1} T}$ and $W_{S r, T}$ indicate the total work needed for the solution of equation (17) $N_{S}$ and $N_{H}$ times respectively. Then

$$
R_{T W}=\frac{W_{D H, T}}{W_{S t, T}}=\frac{2 M W_{D H}+W_{S t}}{N_{S} W_{S t}} .
$$

Considering a $N_{S}=6$ and $N_{H}=13(M=6)$ as the minimum necessary for a comparatle perforrnance of the two LTBE methods (meeting acceptable accuracy crieria), a theoretical estimate of $R_{T W}$ is given in Figure 10 for various computers. It is evident that for the same discretization, the work (and consequently the execution time) for the solution of the coefficient matrix equation (17) in the Dellong LTBE method is at least 12 (and up to slightly over 16) times the work needed for the Stellfest LTBE version. Conversely, Figure 10 indicates that if computer memory is not a limitation, the use of the DeHoog LTBE version may be advantageous only if observations at more than 12-16 times are desired (since equation (17) does not need to be solved for more than one set of $\lambda_{\nu}\left(\nu=1, \ldots, N_{H}\right)$ in the range $\left.[0, T]\right)$. However, in practice this number is higher since the considerable computational effort expended in the computation of the intermeciate complex vectors $\vec{A}, \vec{B}, \vec{d}, \vec{q}, \vec{e}$ is not considered in equation (40).

\section{SUMMARY}

The Laplace Transform Boundary Element (LTBE) method is a recently introduced (Moridis and Reddel [10]) numerical method, and has been used for the solution of diffusion type PDEs. LTBE completely eliminates the time dependency of the problem and the need for time discretization, yielding solutions numerical in space and semianalytical in time. It consists of 4 steps: 1) A Laplace transform is perfor rned on the PDE, 2) the transformed PDE is approximated using the Boundary Element Method (BEM), 
3) the resulting system of simultaneous equations is solved and the transformed vector of unknowns is determined in the Laplace space, and 4) the solution vector obtained in step 3 is inverted numerically to yield the solution in time.

The Stehfest and the DeHoog formulations of LTBE, based on two different numerical inversion algorithrns, are investigated. Both formulations produce comparable, extremely accurate solutions by combining the Laplace space solutions obtained for sets of discrete values of the Laplace parameter $\lambda$. The Stehfest formulation uses real values for the $\lambda_{\nu}\left(\nu=1, \ldots, N_{S}\right)$ parameters, combines linearly the $N_{S}$ solutions without requiring storage of the individual $N_{S}$ solution vectors, but yields a solution at \& single observation time $t$ for each set of $\lambda$. It is simple to code, and yields a very accurate solution for a relatively small $N_{S}(6$ to 8$)$.

The DeHoog formulation uses complex values for the $\lambda_{\nu}\left(\nu=0, \ldots, N_{H}\right)$ parameters, requires storage of the $N_{H}$ individual solution vectors, uses non-linear combinations of the $N_{H}$ solutions, and needs a minimum of $N_{H}=11$ matrix inversions. However, solutions at a range of times $t$ can be obtained from a single set of $\lambda_{1}$. Compared to the Stehfest LTBE, an optimized DeHoog LTBE produces near-singular matrices 4 times as large (by doubling the number of equations and the bandwidth), and increases (1) the execution time requirements per matrix inversion by at least a factor of 12 and (2) the memory requirements by at least a factor of 4 . The Stelifest LTBE seems to have a clear overall advantage, with the exception of cases when the sinks or sources involved are very steep functions of time (i.e. step functicns or spikes).

\section{ACKNOWLEDGEMENTS}

This work was supported by the U.S. Department of Energy under Contract No. DEAC03-76SF00098. The author thanks Dr. Karsien Pruess and Ms. Chris Doughty for their useful review comments.

\section{REFERENCES}

1. Brebbia, C.A. (Ed.). Topics in Boundary Element Research, Vol. 1, Basic Principles and Applications, Springer-Verlag, Berlin and New York, 1984.

2. Liggett, J.A. and Liu, P.L.-F. The Boundary Integral Equation Method for Prorous Media Flow, George Allen Unwin, 1983.

3. Curran, D.A.S., Cross, M. and Lewis, B.A. 'Solution of Parabolic Differential Equations by the Boundary Element Method Using Discretization in Time', Appl. Math. Modelling, Vol. 4, pp. 398-400, 1980.

4. Brebbia, C.A., Telles, J.C.F. and Wrobel, L.C. Boundary Element Techniques, Spinger-Verlag, Berlin and New York, 1984.

5. Wrobel., L.C. and Brebbia, C.A. 'Time Dependent Potential Problems. Chapter 6, Progress in Buundary Elements Methods', (Ed. Brebbia, C.A.), Vol. 1, Pentech Press, London, 1981.

6. Schapery, R. A. 'Approximate Methods of Thansform Inversion for Visco-elastic Stress Analysis', Proc. Fourth U.S. National Congress on Applied Mechanics, Vol. 2, 1962 .

7. Rizzo, F.J. and Shippy, D.H. 'A Method of Solution for Certain Problems of Transient Heat Conduction', AlAA J., Vol. 8, 11, pp. 2004-2009, 1970.

8. Liggett, J.A and Liu, P.L.-F. 'Unsteady Flow in Confined Aquifers: A Comparison of 'Two Boundary Integral Meshods', Water Ressources Research, Vol. 15, pp. 861$866,1979$.

9. Lachat, J.C. and Combescure, A. 'Laplace Transform and Boundary Integral Equa- 
tion: Application to Trarisient Heat Conduction Problems', Innovarive Numerical Analysis in Applied Engineering Science (Eds. Cruse, T.A. et al.), CETIM, Versailles, 1877 .

10. SIoridis, G.J. and Reddell, D.L. 'The Laplace Transform Boundary Element (LTBE) Numerical Method for the Solution of Diffusion-Type Equations', Boundary Elements XIII (Ed. Brebbia C. A. and Gibson, G. S.), pp. 83-97, Proceedings of the 13th International Conference on Boundary Element Methods, Tulsa, 1991. Computational Mechanics Publications and Elsevier Applied Sciences, London, 1991.

11. Moridis, G.J. and Reddell, D.L. "The Laplace Trasisform Finite Difference (LTFD) Metbod for Simulation of Flow Through Porous Media', Water Resour. Res., vol. $27,8, \mathrm{pp} .1873-1884,1991$.

12. Moridis, G.J. and Reddell, D.L. 'The Laplace Transform Finite Difference (LTFD) Numerical Method for the Simulation of Solute 'Transport in Groundwater', paper No. H42A-7, 1990 AGU Fall Meeting, San Fransisco, Nec. 3-7, EOS Trans. of the AGU, 71(43), 1990.

13. Moridis, G.j. and Reddell, D.L. 'The Laplace Transform Finite Element, (LTFE) Numerical Method for the Solution of the Groundwater Equation', paper Y22C-4, AGU 91 Spring Meeting, Baltimore, May 28-31, 1991, EOS Trans. of the AGU, $72(17), 1921$.

14. Moridis, G.J. McVay, D. A., Reddell, D. L. and Biastitgame, T. A., 'The Laplace Transform Finite Difference (LTFD) Method for the Simulavion of Compressible Fluid Flow in Reservoirs', Paper 22888, Proceedings, Society of Petroleum Engineers 66th Annual Technical Conference, Dallas, Texas, Octuber 6-9, 1991

15. Greenberg, M.D. Applications of Green's Functions in Science and Engineering, Prentice Hall, Englewood Cliffs, 1971.

16. A.bramowitz, M. and Stegun, I.A. (Eds.). Handbook of Mathematical Functions, Dover, New York, 1965.

17. Stehfest, H. 'Nume ical Inversion of Laplace Transforms', J. ACM, Vol. 13, pp. $47-49,1970$.

18. Stehfest, H. 'Numerical Inversion of Laplace Transforms, J. ACM, Vol. 13, pp. 56, 1970.

19. DeHoog, F. R., Knight, J. H. and Stokes, A. N. 'An Improved Method for Numerical Inversicn of Laplace 'Transforms', SIAM J. Sci. Stat. Comput., vol. 3, 3, pp. 35\%$366,1982$.

20. Sudicky, E. A. "The Laplace Transform Galerkin Technique: A Time-Continuous Finite Element 'Theory and Application to Mass 'Transport in Ground water', Water Resour. Res., vol. 25, 8, pp.1833-1846, 1989.

21. Crump, K. S., 'Numerical Inversion of Laplace Transforms Using a Fourier Series Approximation', J. Assoc. Comput. Mach., vol 23, 1, pp. 89-96, 1976.

22. Theis, C. V.. 'The relation Between the Lowering of the Piezometric Surface and the Rate and Duration of Discharge of a Well Using Groundwater Storage', Trans. Am. Geophys. Un., vol. 16, pp. 519-524, 1935. 


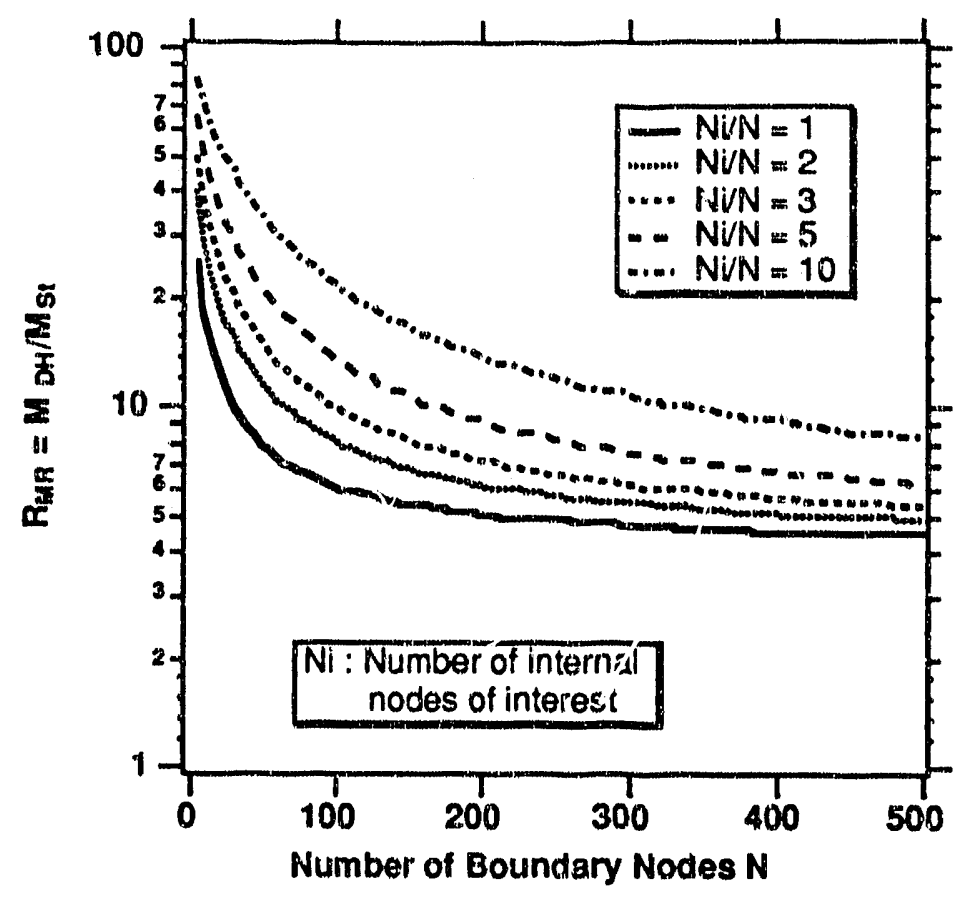

Fig. 1. Ratio of computer memory requirements of the two LTBE formulations $(M=5)$.

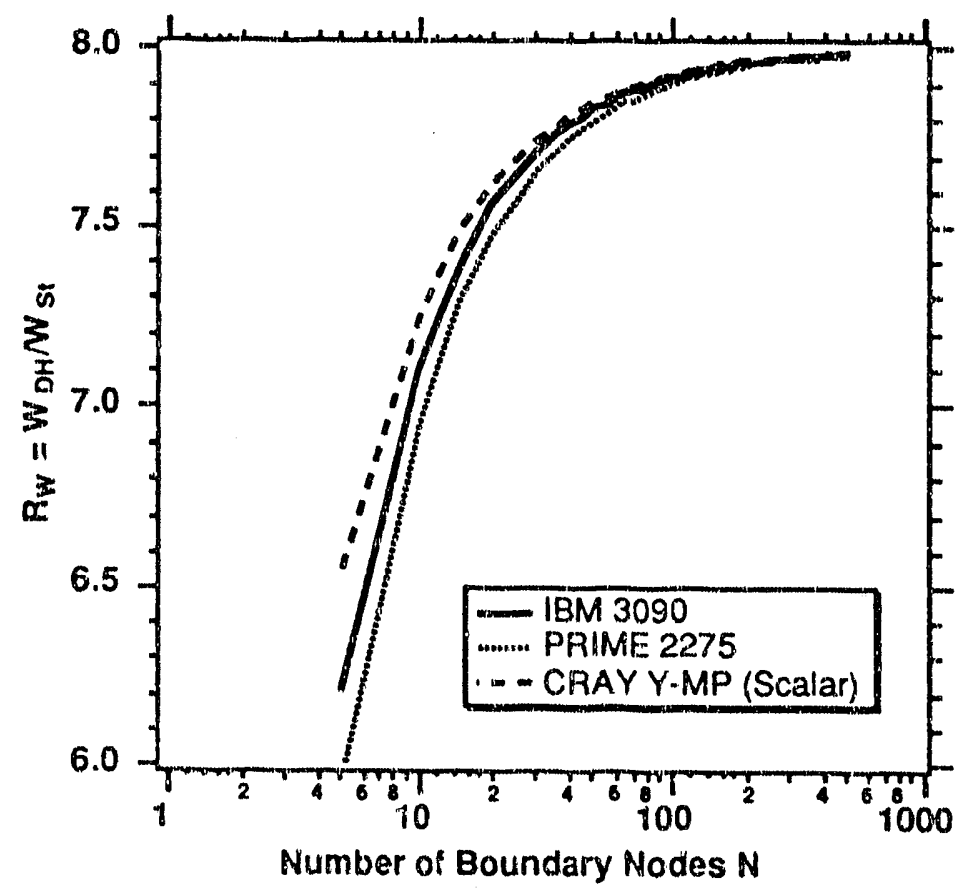

Fig. 2. Work ratio of the two LTBE formulations for various computers $(M=5)$. 

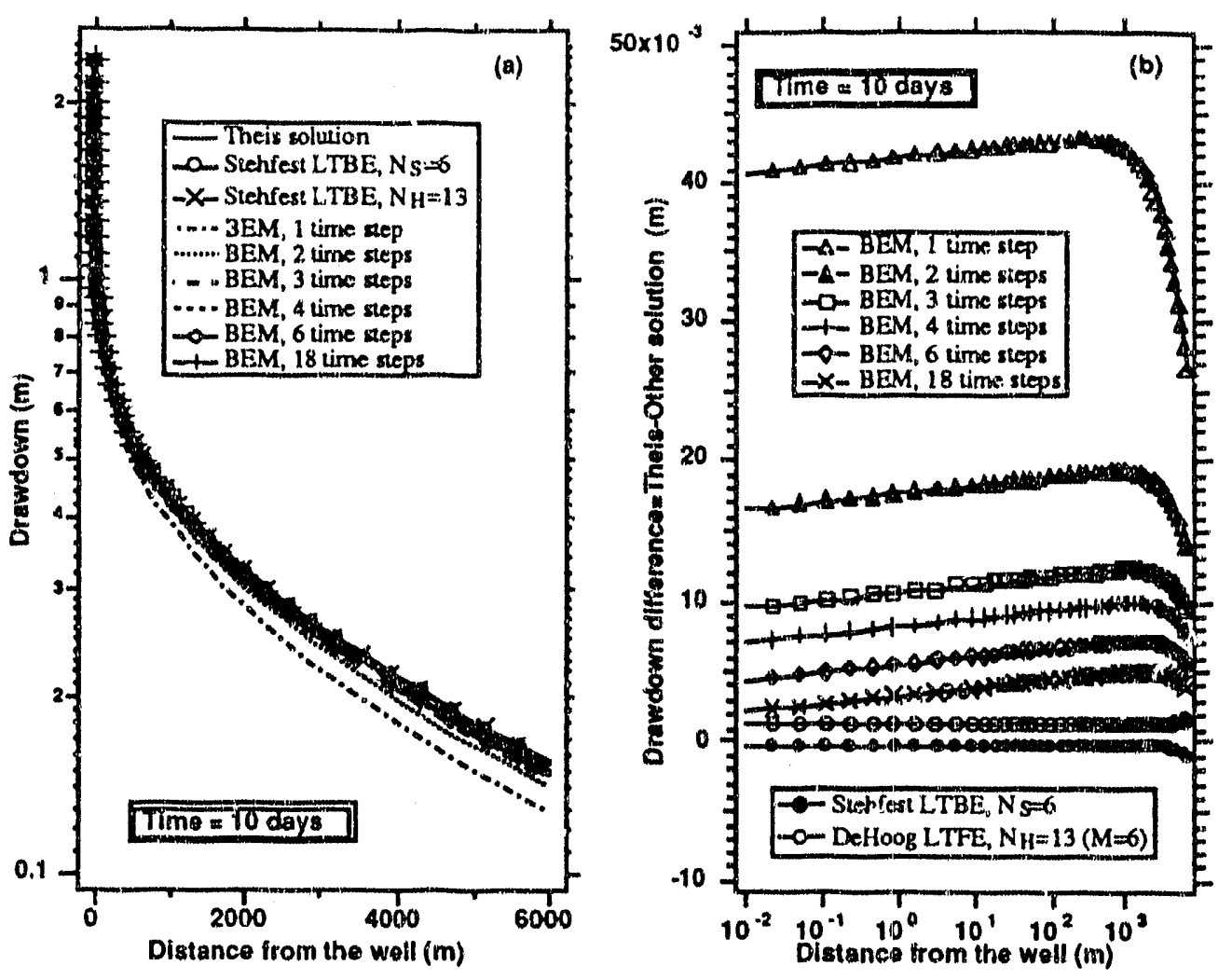

Fig. 3. Drawdown (a) and \% drawdown difference from the analytical solution (b) obtained from a standard BEM and the two ITBE methods.

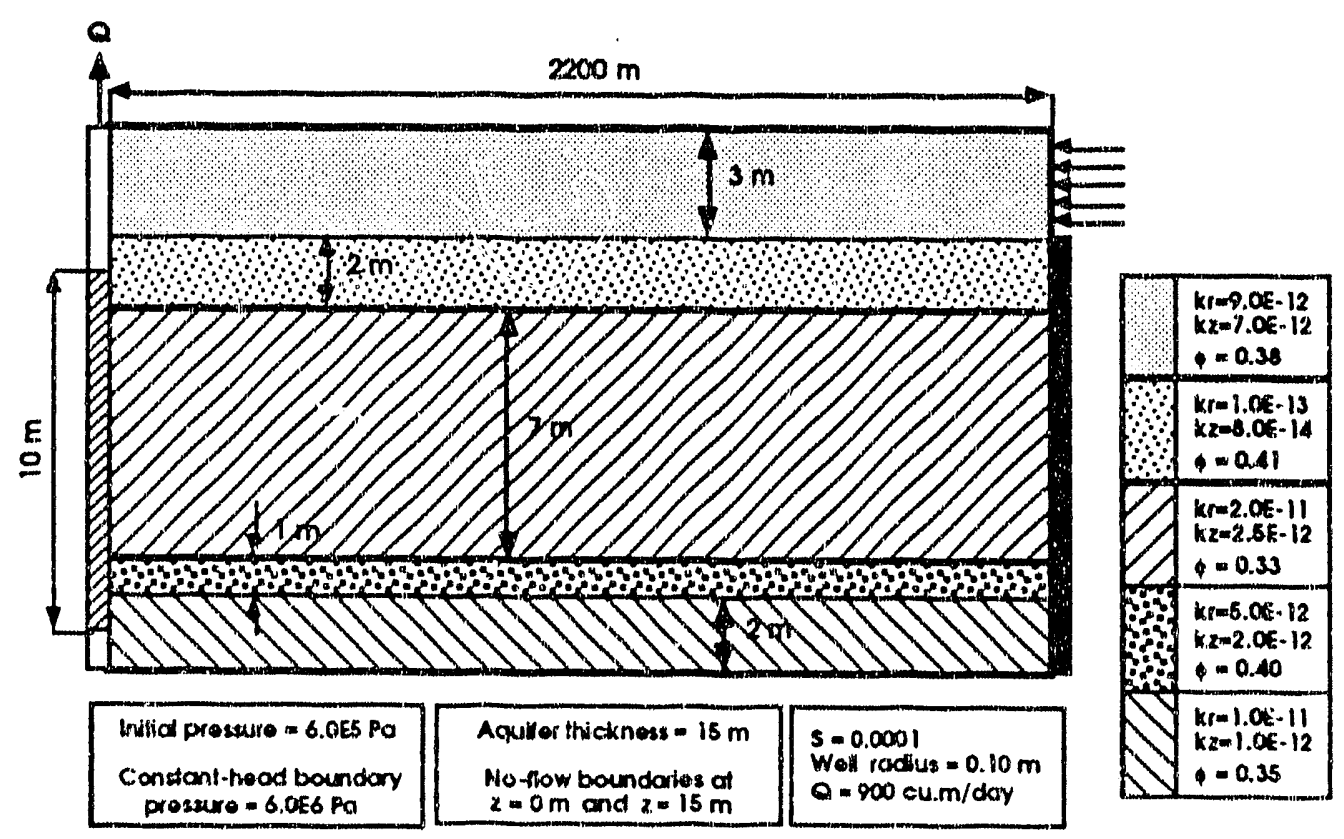

Fig. 4. Geometry, boundaries, and properties of the aquifer in Test Case 2. $k_{r}$ and $k_{z}$ are in $m^{2}$. 


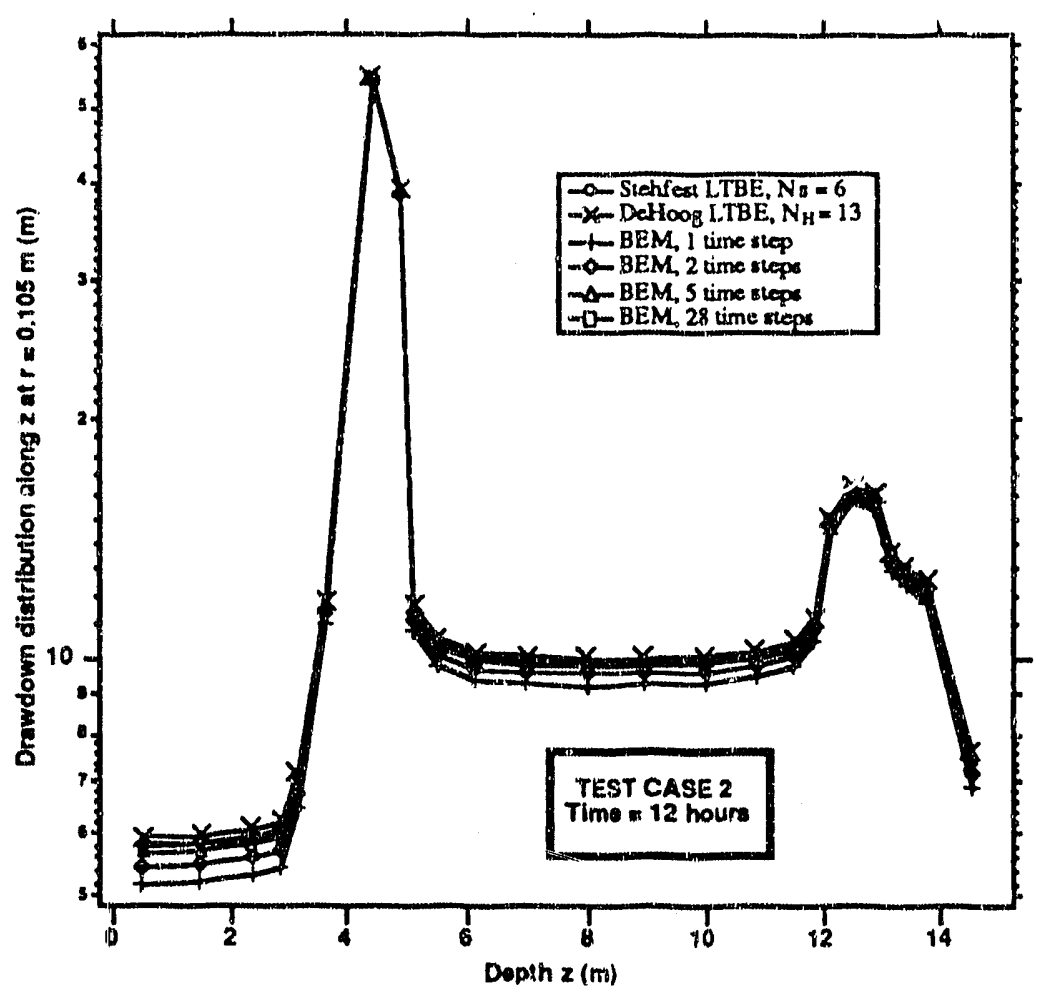

Fig. 5. Test Case 2: drawdown distribution from BEM and the two LTBE methods.

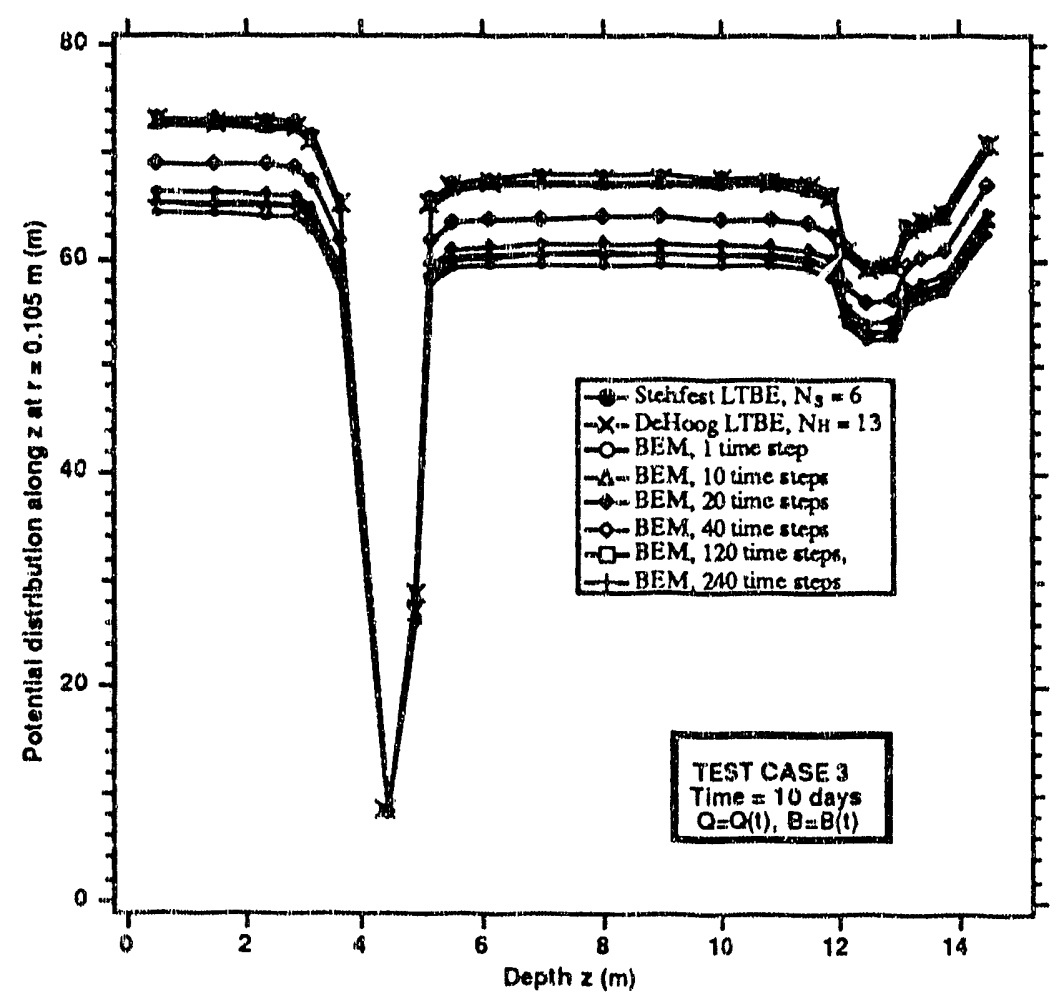

Fig. 6. Test Case 3: potential (head) distribution from BEM and the two LTBE methods. 


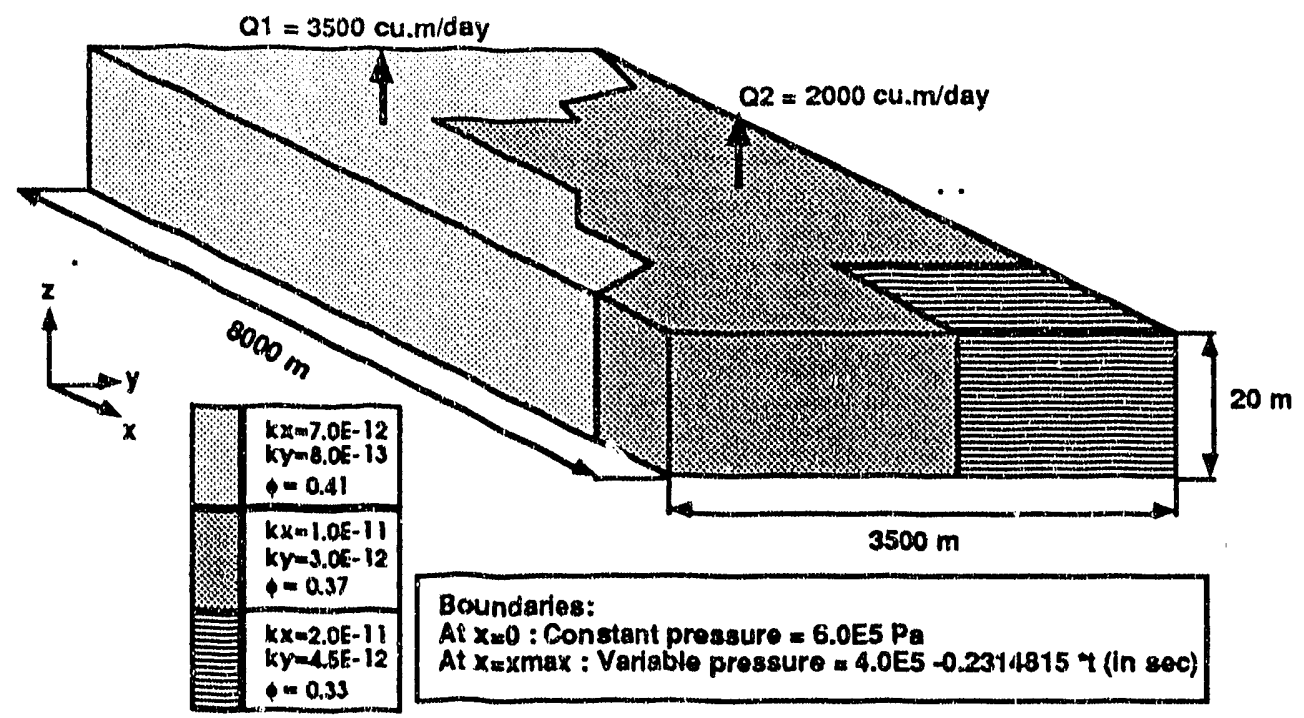

Fig. 7. Geometry, boundaries, and properties of the aquifer in Test Case 4. $k_{x}, k_{y}$ and $k_{z}$ are in $m^{2}$.

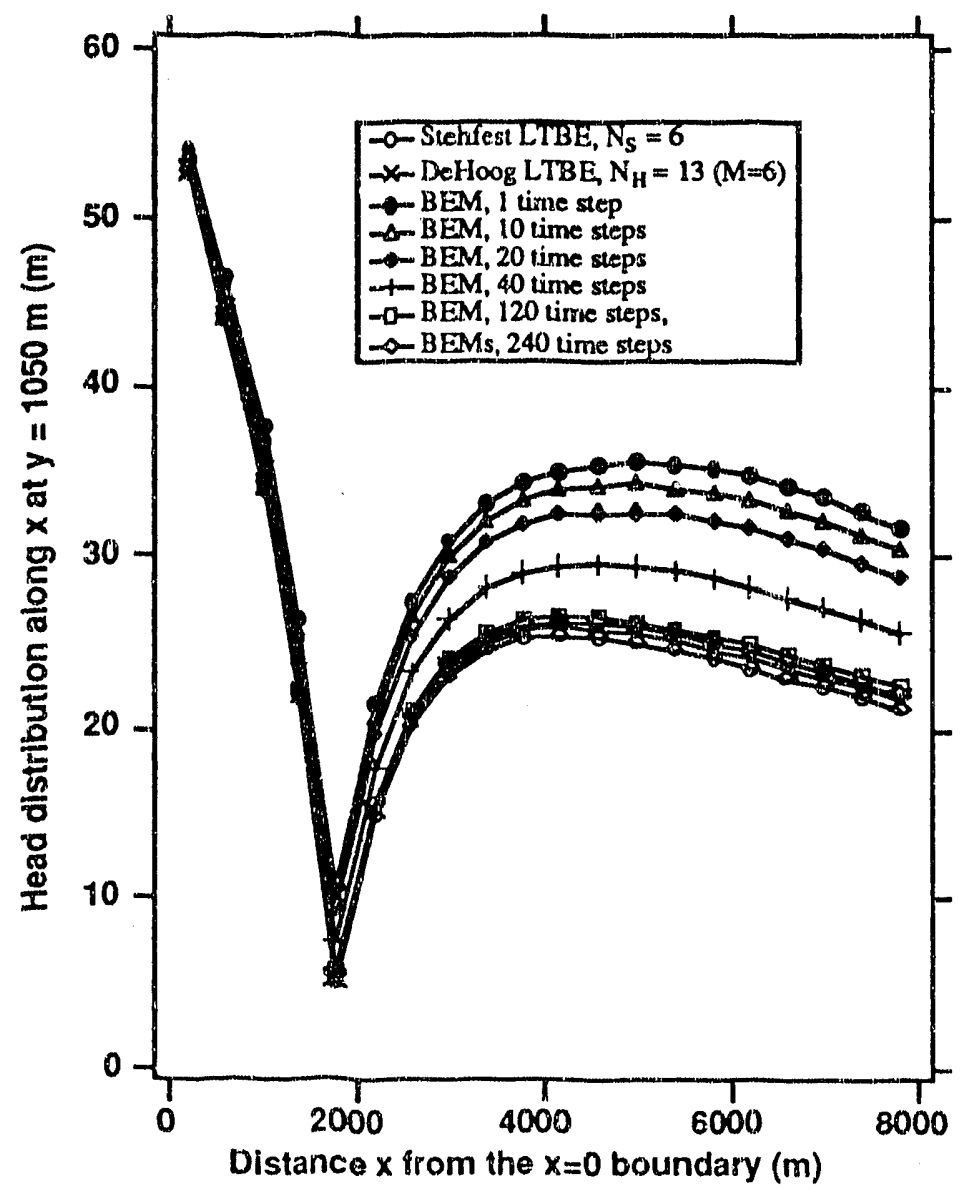

Fig. 8. Test Case 4: potential (head) distribution obtained from a standard BEM and the two LTBE methods. 


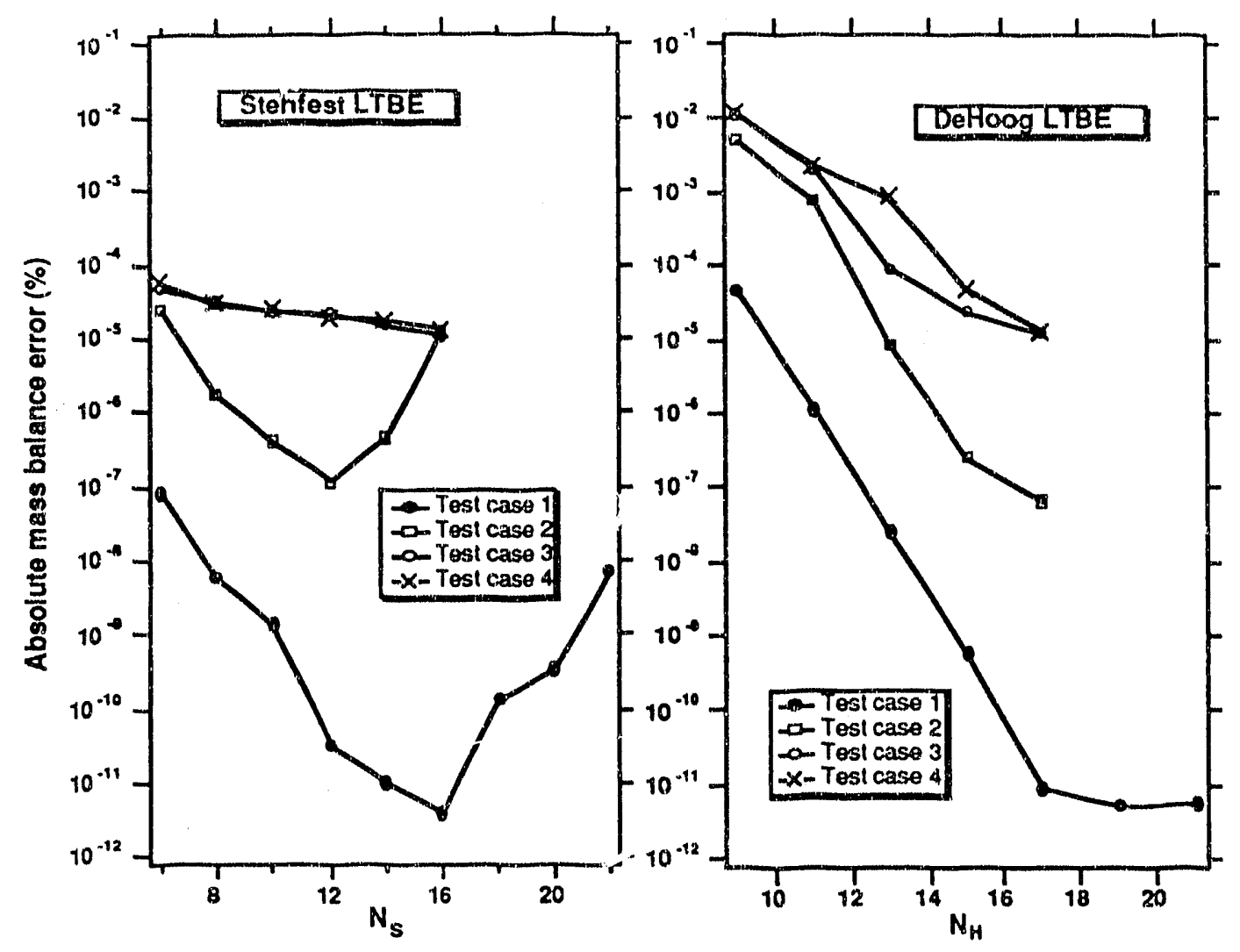

Fig. 9. Mass balance error of the two LTBE schemes.

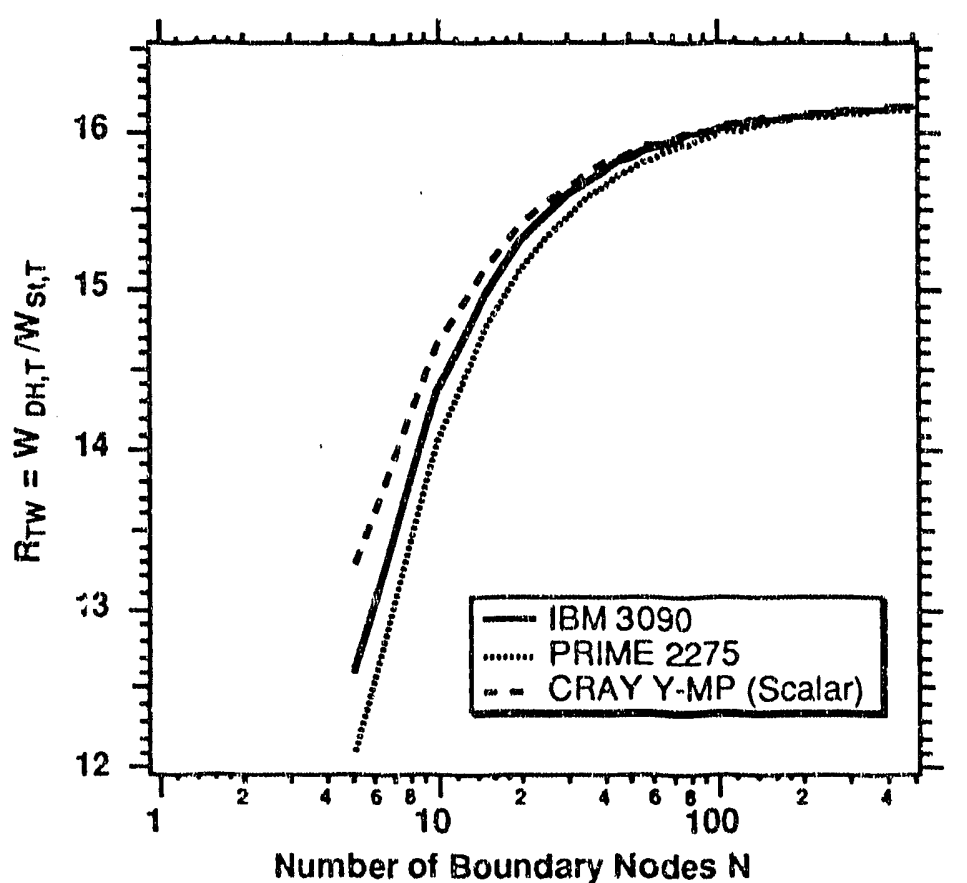

Fig. 10. Ratio of stal work ratio of the two L'TBE formulations for various computers $\left(N_{S}=6\right.$ and $\left.N_{H}=13\right)$. 

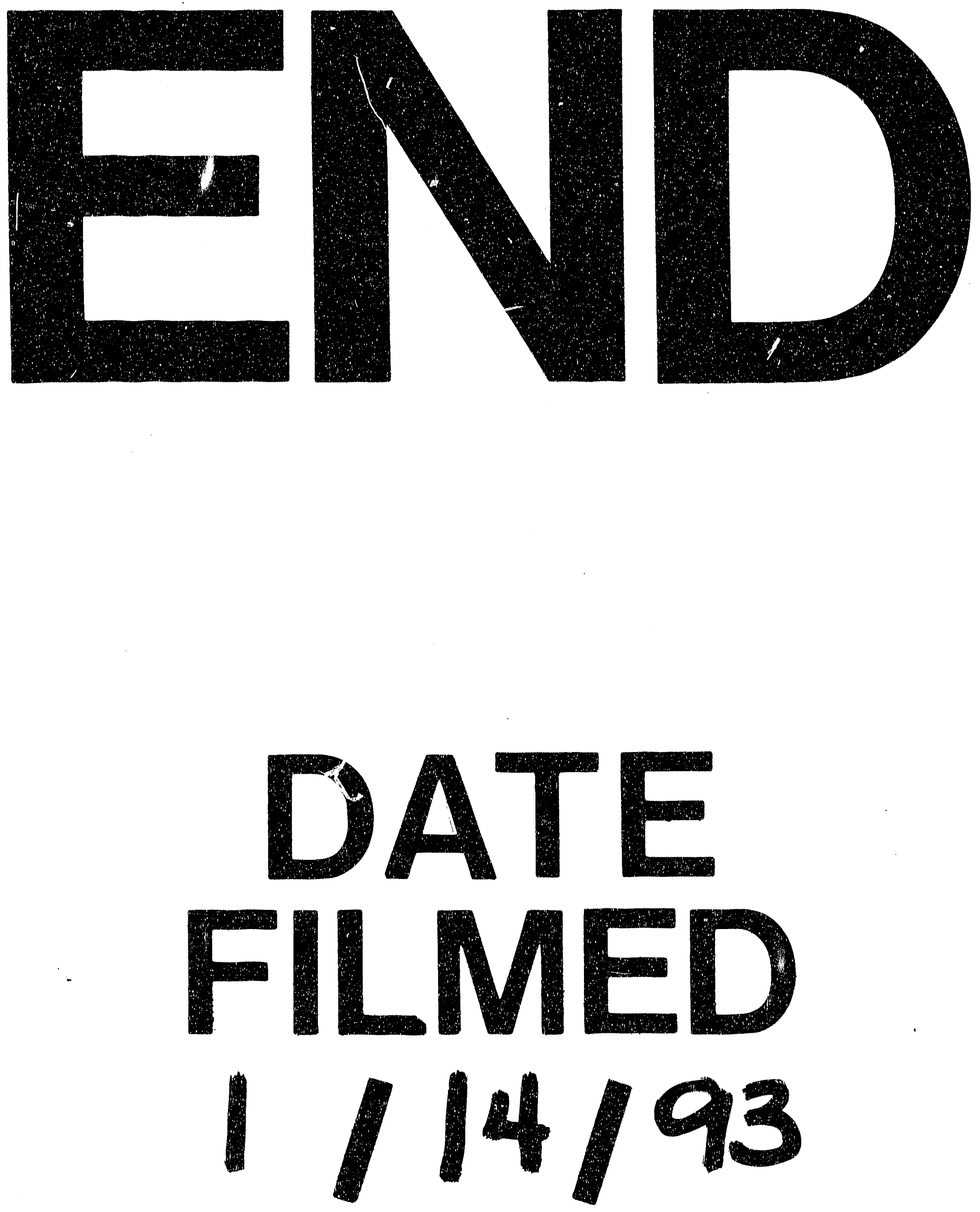
